\title{
Improving Estimates for Differential Operators in Two Independent Variables
}

\author{
By \\ Humio SUzUKI*
}

\section{$\S 1$. Introduction}

Let $P(x, D)$ be a differential operator of order $m$ with $C^{\infty}$ coefficients defined in an open subset $\Omega$ of $R^{n}$. We shall say that an improving estimate for $P$ holds in $\Omega$, if the following condition is fulfilled:

For every compact set $K$ in $\Omega$, there exists a positive number $\rho>0$ such that

$$
\|u\|_{m-1+\rho} \leqq C\left(\|P u\|_{0}+\|\left. u\right|_{m-1}\right), \quad u \in C_{0}^{\infty}(K),
$$

where $\|u\|_{s}$ is the norm of the space $H_{s}[1, \S 2.4]$.

In this paper we shall study conditions for the validity of improving estimates, when the number of independent variables is $t w o$.

We denote the principal symbol of $P(x, D)$ by $p(x, \xi)$. We define the $k$ th commutator $c_{p}^{k}(x, \xi)$ of $p(x, \xi)$ by induction:

$$
\begin{aligned}
& c_{p}^{0}(x, \xi)=p(x, \xi), \text { the complex conjugate of } p(x, \xi), \\
& c_{p}^{k}(x, \xi)=\left[p, c_{p}^{k-1}\right],
\end{aligned}
$$

where $[p, q]$ is the Poisson bracket of $p$ and $q$,

$$
[p, q]=\sum_{i=1}^{n}\left(\frac{\partial p}{\partial \xi_{i}} \frac{\partial q}{\partial x_{i}}-\frac{\partial p}{\partial x_{i}} \frac{\partial q}{\partial \xi_{i}}\right)
$$

Let $k_{p}(x, \xi)$ denote the first value of $k$ for which $c_{p}^{k}(x, \xi) \neq 0$. If $c_{p}^{k}(x, \xi)$ vanishes for all values of $k$, we define $k_{p}(x, \xi)$ to be $\infty$.

Our main result is the following

Received October 15, 1969.

Communicated by S. Matsuura.

* Department of Mathematics, Tokyo University of Education. 
Theorem. Let $P(x, D)$ be a differential operator with $C^{\infty}$ coefficients defined in an open set $\Omega$ in the two dimensional space $R^{2}$. Assume that the real characteristics of $P$ are simple, that is, $p(x, \xi)=0(\xi \neq 0)$ implies $\frac{\partial p(x, \xi)}{\partial \xi_{i}} \neq 0$ for some $i$. Then the following is a sufficient condition for the validity of an improving estimate for $P$ in $\Omega$ :

(A) For every $x \in \Omega$, and every $\xi \in R^{2}(\xi \neq 0), k_{p}(x, \xi)$ is finite and even.

If the coefficients of the principal part of $P$ are analytic, (A) is also a necessary condition for the improving estimate.

Remark. We can easily extend the results of this paper to determined systems of differential operators of type $(s, t)$ in the sense of Douglis and Nirenberg for which the determinant of the principal parts satisfies the hypothesis we have made in the case of one equation. It is also clear that the results given here can be extended to two-dimensional manifolds, since everything is invariant under diffeomorphisms.

\section{$\S 2 . \quad$ Preliminaries}

We shall first list some elementary facts concerning improving estimates and the condition (A).

$1^{\circ}$. The validity of an improving estimate is a local property. Application of a partition of unity shows that an improving estimate holds in $\Omega$, if it is valid for some neighborhood of any point in $\Omega$.

$2^{\circ}$. From the invariance of the spaces $H_{s}$ under diffeomorphisms, it follows that the validity of an improving estimate is an invariant property.

$3^{\circ}$. Lower order terms are irrelevant for the validity of an improving estimate.

$4^{\circ}$. Since the values of the Poisson bracket do not depend on the choice of local coordinates, the values of $k_{p}(x, \xi)$ are invariant under a change of variables, and so is the condition (A).

$5^{\circ}$. The validity of an improving estimate is equivalent to the following conditions [2,' $\S 1.0$ and 1.4$]$.

(a) For every compact set $K$ in $\Omega$, there is a positive number $\rho>0$ such that for every real number $s$, we have an estimate 


$$
\|u\|_{s+m-1+\rho} \leqq C_{s}\left(\|P u\|_{s}+\|u\|_{s+m-1}\right), \quad u \in C_{0}^{\infty}(K) .
$$

(b) For every compact set $K$ in $\Omega$, there is a positive number $\rho>0$ such that for every real number $s$, $u \in \mathcal{E}^{\prime}(K), u \in H_{s+m-1}$ and $P u \in H_{s}$ implies $u \in H_{\boldsymbol{s}+m-1+\rho}$.

(c) For every relatively compact open set $\omega$ in $\Omega$, there is a positive number $\rho>0$ such that for every real number $s$,

$$
u \in \mathscr{D}^{\prime}(\omega) \text { and } P u \in H_{s}^{\text {loc }}(\omega) \text { implies } u \in H_{s+m_{-1+\rho}}^{\text {loc }}(\omega) \text {. }
$$

In particular,

$$
u \in \mathscr{D}^{\prime}(\Omega) \text { and } P u \in C^{\infty}(\Omega) \text { implies } u \in C^{\infty}(\Omega) .
$$

We shall now study the condition (A) for a product of operators. The main point is the following proposition.

Proposition 2.1. If $p(x, \xi)$ and $q(x, \xi)$ are $C^{\infty}$ functions of $x$ and $\xi$, then

$$
k_{p q}(x, \xi) \geqq k_{p}(x, \xi) .
$$

If, in particular, $q(x, \xi) \neq 0$, then $k_{p q}(x, \xi)=k_{p}(x, \xi)$.

Proof. The Poisson bracket $[p, q]$ satisfies the following relation

$$
[p q, r]=p[q, r]+q[p, r] \text {. }
$$

Using this relation we have

$$
\begin{aligned}
c_{p q}^{0} & =\bar{p} \bar{q}=c_{p}^{0} \bar{q}, \\
c_{p q}^{1} & =p \bar{p}[q, \bar{q}]+p \bar{q}[q, \bar{p}]+q \bar{p}[p, \bar{q}]+q \bar{q}[p, \bar{p}] \\
& =p c_{p}^{0}[q, \bar{q}]+p \bar{q}[q, \bar{p}]+q c_{p}^{0}[p, \bar{q}]+q \bar{q} c_{p}^{1} .
\end{aligned}
$$

By induction, we see that the $k$ th commutator $c_{p q}^{k}$ of $p q$ is a sum of terms each of which is a product of $k+2$ factors, and at least one of the factors is equal to one of the quantities $p, c_{p}^{0}, c_{p}^{1}, \cdots, c_{p}^{k}$. Hence $c_{p q}^{k}=0$, if $k<k_{p}(x, \xi)$. This proves the inequality (2.1).

Next assume that $p(x, \xi)$ is a product

$$
p(x, \xi)=p_{1}(x, \xi) \cdots p_{\mu}(x, \xi) .
$$

If the real characteristics of $p(x, \xi)$ are simple, then for every $(x, \xi)$, there is at most one $i$ such that $p_{i}(x, \xi)=0$. Furthermore the real characteristics of each $p_{i}(x, \xi)$ are simple. 
Proposition 2.2. Assume that the real characteristics of $p(x, \xi)$ are simple and $p(x, \xi)=p_{1}(x, \xi) \cdots p_{\mu}(x, \xi)$. Then the condition $(A)$ holds for $p$, if and only if, for every $i$, the condition $(A)$ holds for $p_{i}$.

Proof. Necessity. For every $i$, and every $(x, \xi), p_{i}(x, \xi) \neq 0$ or $q_{i}(x, \xi)=p_{1} \cdots p_{i-1} p_{i+1} \cdots p_{\mu} \neq 0$. If $p_{i}(x, \xi) \neq 0$, then $k_{p_{i}}(x, \xi)=0$. If $q_{i}(x, \xi) \neq 0$, then, by Prop. 2.1, $k_{p_{i}}(x, \xi)=k_{p}(x, \xi)$. Hence, in either case, $k_{p_{i}}(x, \xi)$ is finite and even.

Sufficiency. For every $(x, \xi)$, there is an $i$ such that $q_{i}(x, \xi) \neq 0$. By Prop. 2.1, $k_{p}(x, \xi)=k_{p_{i}}(x, \xi)$. Hence $k_{p}(x, \xi)$ is finite and even.

Since the problem is local, we need only consider what happens in the neighborhood of a given point $\left(x_{0}, y_{0}\right)$ of $\Omega$. From now on we denote the variables by $x, y, \xi, \eta$ instead of $x, \xi$. After a change of variables, we may assume that the coefficient of $\xi^{m}$ in $p(x, y ; \xi, \eta)$ does not vanish in a neighborhood of $x_{0}=y_{0}=0$. If the real characteristics of $\dot{p}$ are simple, we can write, in a neighborhood of $O=(0,0)$,

$$
p=p_{1} \cdots p_{\mu} q, \quad p_{i}=\xi-\lambda_{i}(x, y) \eta .
$$

Here $\lambda_{i}(x, y)$ are $C^{\infty}$ functions such that $\lambda_{i}(x, y) \neq \lambda_{j}(x, y)$ if $i \neq j$, and $q$ is an elliptic polynomial of degree $m-\mu$ with $C^{\infty}$ coefficients. If the coefficients of $p$ are analytic, the coefficients of $p_{i}$ and $q$ are also analytic.

\section{$\S 3$. Sufficiency of the Condition $(A)$ : Reduction to the First Order Case}

Let $P\left(x, y ; D_{x}, D_{y}\right)$ be a differential operator of order $m$ with simple real characteristics defined in a neighborhood $\Omega$ of $O$. Here $D_{x}=\partial / \partial x, D_{y}=\partial / \partial y$. We assume that $p(x, y ; \xi, \eta)$ has the form (2.2). If the condition (A) holds for $p$, then it also holds for each $p_{i}$. We shall show in this section that an improving estimate holds for $P$ in $\Omega$, if it is valid for each $p_{i}\left(x, y ; D_{x}, D_{y}\right)$.

We denote by $\Pi$ the product of the polynomials $p_{1}, \cdots, p_{\mu}$ :

$$
\Pi(x, y ; \xi, \eta)=p_{1} \cdots p_{\mu}=\xi^{\mu}+\sum_{k=1}^{\mu} a_{k}(x, y) \xi^{\mu-k} \eta^{k} .
$$

For $u \in C_{0}^{\infty}(\Omega)$, let $U(x, y)$ be the vector valued function with com- 
ponents $u_{k}(x, y)=D_{x}^{k-1} D_{y}^{\mu-k} u(x, y), k=1, \cdots, \mu$. If we write $F$ for $\left(0, \cdots, 0, \Pi\left(x, y ; D_{x}, D_{y}\right) u\right)$, and if $A$ denotes the matrix of entries $a_{i j}=1$ if $j=i+1, a_{\mu_{j}}=-a_{\mu_{-j+1}}$ and remaining ones equal zero, then we have

$$
D_{x} U-A D_{y} U=F
$$

Let $N^{\prime}$ be the matrix with entries $n_{i j}^{\prime}=\lambda_{j}^{i-1}$. Then, since the $\lambda_{i}$ are all distinct, $N^{\prime}$ is nonsingular. If we denote by $\Lambda$ the diagonal matrix with entries $\lambda_{i}$ in the main diagonal, then $N A=\Lambda N$ with $N=\left(N^{\prime}\right)^{-1}$. Hence, setting $V=N U$, we have

$$
D_{x} V-\Lambda D_{y} V=N F+\left(D_{x} N-\Lambda D_{y} N\right) U .
$$

We use the notation $\|U\|_{s}^{2}=\sum_{k=1}^{\mu}\left\|u_{k}\right\|_{s}^{2}$. If an improving estimate is valid for each $p_{i}$, then for every compact set $K$ in $\Omega$, there is a $\rho>0$ such that

$$
\|V\|_{\rho}^{2} \leqq C\left(\left\|D_{x} V-\Lambda D_{y} V\right\|_{0}^{2}+\|V\|_{0}^{2}\right), \quad V \in C_{0}^{\infty}(K)^{\mu} .
$$

Since $\|U\|_{s}$ is equivalent to $\|V\|_{s}$ for $U \in C_{0}^{\infty}(K)^{\mu}$, we have, with a new constant $C$,

$$
\|U\|_{\rho}^{2} \leqq C\left(\|F\|_{0}^{2}+\|U\|_{0}^{2}\right), \quad u \in C_{0}^{\infty}(K) .
$$

When $u \in C_{0}^{\infty}(K),\|U\|_{s}$ is equivalent to $\|u\|_{\mu_{-1+s}}$, hence

$$
\|u\|_{\mu_{-1+\rho}} \leqq C\left(\|\Pi u\|_{0}+\|u\|_{\mu_{-1}}\right), \quad u \in C_{0}^{\infty}(K) .
$$

Since the operator $q\left(x, y ; D_{x}, D_{y}\right)$ is elliptic of order $m-\mu$, we have

$$
\|u\|_{m-1+\rho} \leqq C\left(\|q u\|_{\mu_{-1+\rho}}+\|u\|_{m-1}\right), \quad u \in C_{0}^{\infty}(K) .
$$

Combining (3.1) and (3.2),

$$
\|u\|_{m-1+\rho} \leqq C\left(\|\Pi q u\|_{0}+\|u\|_{m-1}\right), \quad u \in C_{0}^{\infty}(K) .
$$

Since $\Pi q-P$ is an operator of order $\leqq m-1$, we obtain an improving estimate for $P$.

\section{$\S 4$. Sufficiency of the Condition $(A)$ : The First Order Case}

Let $P$ be a first order differential operator of the form

$$
P=D_{x}-\lambda(x, y) D_{y},
$$


where $\lambda(x, y)$ is a $C^{\infty}$ function in a neighborhood of $O$. We have to prove that an improving estimate holds, if $P$ satisfies the condition (A).

We shall first bring the differential operator to a suitable form. It is possible to introduce new local coordinates in a neighborhood of $O$, so that the operator takes the form

$$
P=a(x, y)\left(D_{x}+i b(x, y) D_{y}\right),
$$

where $a(x, y)$ is a non-vanishing complex valued $C^{\infty}$ function, and $b(x, y)$ is a real-valued $C^{\infty}$ function [4]. Since $k_{p}(x, y ; \xi, \eta)$ is invariant under multiplication of $P$ by a non-vanishing function, we may suppose that $P$ has the form

$$
P=D_{x}+i b(x, y) D_{y},
$$

where $b(x, y)$ is a real-valued $C^{\infty}$ function defined in a neighborhood $\Omega=\left\{(x, y)|| x|| y \mid,<\delta_{0}\right)$ of $O$.

For the differential operator $P$ of the form (4.1), $c_{p}^{b}$ becomes

$$
c_{p}^{k}=\left(-2 i \frac{\partial^{k} b}{\partial x^{k}}+\sum_{j=0}^{k-1} c_{j k} \frac{\partial^{j} b}{\partial x^{j}}\right) \eta, \quad k \geqq 1,
$$

where $c_{j k}$ are $C^{\infty}$ functions depending on $b(x, y)$. If we denote by $k_{p}(x, y)$ the first value of $k$ such that

$$
\frac{\partial^{k} b(x, y)}{\partial x^{k}} \neq 0,
$$

then the condition (A) is equivalent to the following condition:

$\left(\mathrm{A}^{\prime}\right)$ At every point $(x, y)$ of $\Omega, k_{p}(x, y)$ is finite and even. It follows therefore that, if the condition (A) holds, the sign of the function $b(x, y)$ does not vary with $x$ and $y$. We may thus suppose that $b(x, y) \geqq 0$.

Now we shall construct a left parametrix for the differential operator $P$ of the form (4.1). Let $E$ be an integral operator

$$
(E f)\left(x^{\prime}, y^{\prime}\right)=\int e\left(x^{\prime}, y^{\prime} ; x, y\right) f(x, y) d x d y
$$

with the kernel

$$
e\left(x^{\prime}, y^{\prime} ; x, y\right)=\left\{2 \pi\left[\int_{x}^{x^{\prime}} b\left(z, y^{\prime}\right) d z+i\left(y^{\prime}-y\right)\right]\right\}^{-1} .
$$


It follows from $\left(\mathrm{A}^{\prime}\right)$ that $e$ is a $C^{\infty}$ function outside the diagonal in $\Omega \times \Omega$.

For simplicity we write $X$ and $d X$ instead of $(x, y)$ and $d x d y$.

Proposition 4.1. If $\omega$ is a sufficiently small neighborhood of $O$, then for every $\rho, 0 \leqq \rho<\left(k_{p}(0,0)+1\right)^{-1}$, there is a constant $M_{\rho}$ such that

$$
\begin{aligned}
& \sup _{X \in \omega} \int_{\omega}\left|e\left(X^{\prime} ; X\right)\right|^{1+\rho} d X^{\prime} \leqq M_{\rho}, \\
& \sup _{X^{\prime} \in \omega} \int_{\omega}\left|e\left(X^{\prime} ; X\right)\right|^{1+\rho} d X \leqq M_{\rho} .
\end{aligned}
$$

Furthermore, for every $\rho, 0 \leqq \rho \leqq 1$, there is a constant $N_{\rho}$ such that if $X, X^{\prime}, X^{\prime \prime} \in \omega$, we have

$$
\begin{aligned}
\left|e\left(X^{\prime} ; X\right)-e\left(X^{\prime \prime} ; X\right)\right| \leqq N_{\rho} \mid & X^{\prime}-X^{\prime \prime}||^{\rho}\left(\left|e\left(X^{\prime} ; X\right)\right|^{1+\rho}\right. \\
& \left.+\left|e\left(X^{\prime \prime} ; X\right)\right|^{1 / \rho}\right) .
\end{aligned}
$$

Proof. If we set $x^{\prime}=x+s, y^{\prime}=y+t$, we have

$$
e^{-1}(x+s, y+t ; x, y)=2 \pi\left[\int_{x}^{x+s} b(z, y+t) d z+i t\right] .
$$

We can write $b(z, y+t)-b(z, y)=a(z, y, t) t$, where $a$ is a real-valued $C^{\infty}$ function, so that

$$
e^{-1}=2 \pi\left[\int_{x}^{x+s} b(z, y) d z+\left(\int_{x}^{x / s} a(z, y, t) d z+i\right) t\right] .
$$

Hence there is a constant $C$ such that

$$
|e(x+s, y+t ; x, y)| \leqq C(|t|+|B|)^{-1},
$$

where we have written

$$
B(s, x, y)=\int_{x}^{x+s} b(z, y) d z .
$$

If we set $x=y=0$, we have

$$
B(s, 0,0)=\int_{0}^{s} b(z, 0) d z .
$$

Hence $B(s, 0,0)$ has a zero of order exactly $k+1$ at $s=0$, where $k=k_{p}(0,0)$. It follows from the preparation theorem for differentiable functions [3] that for sufficiently small $|s|,|x|,|t|$, say $<\delta$,

$$
B(s, x, y)=C(s, x, y) D(s, x, y)
$$


where $C(s, x, y)$ is a non-vanishing $C^{\infty}$ function, and $D(s, x, y)$ is a distinguished polynomial in $s$ of degree $k+1$ with coefficients $C^{\infty}$ functions of $x$ and $y .{ }^{1)}$

Now we have for every $X=(x, y)$ in $\omega=\{(x, y)|| x|| y \mid,<\delta / 2\}$,

$$
\begin{aligned}
\int_{\omega}\left|e\left(X^{\prime} ; X\right)\right|^{1+\rho} d X^{\prime} & \leqq \int_{-\delta}^{\delta} \int_{-\delta}^{\delta}|e(x+s, y+t ; x, y)|^{1+\rho} d s d t \\
& \leqq C^{1+\rho} \int_{-\delta}^{\delta} \int_{-\delta}^{\delta} \frac{d s d t}{(|t|+|B|)^{1+\rho}} \\
& \leqq C^{1+\rho} \frac{2}{\rho} \int_{-\delta}^{\delta} \frac{d s}{|B|^{\rho}}, \quad \text { if } \rho>0
\end{aligned}
$$

If $\mu_{1}(x, y), \cdots, \mu_{k+1}(x, y)$ are the roots of the polynomial $D(s, x, y)$, we have

$$
\int_{-\delta}^{\delta} \frac{d s}{|B|^{\rho}} \leqq C^{\prime} \int_{-\delta}^{\delta} \frac{d s}{\left|s-\mu_{1}\right|^{\rho} \cdots\left|s-\mu_{k+1}\right|^{\rho}} .
$$

Hence, using Hölder's inequality, we obtain

$$
\int_{-\delta}^{\delta} \frac{d s}{|B|^{\rho}} \leqq C^{\prime}\left(\int_{-\delta}^{\delta} \frac{d s}{\left|s-\mu_{1}\right|^{(k+1) \rho}}\right)^{1 /(k+1)} \cdots\left(\int_{-\delta}^{\delta} \frac{d s}{\left|s-\mu_{k+1}\right|^{(k+1) \rho}}\right)^{1 /(k+1)} .
$$

If $(k+1) \rho<1$, these integrals are convergent. Furthermore

$$
\begin{aligned}
\int_{-\delta}^{\delta} \frac{d s}{\left|s-\mu_{i}(x, y)\right|^{(k+1) \rho}} & \leqq \int_{-\delta}^{\delta} \frac{d s}{\left|s-\operatorname{Re} \mu_{i}(x, y)\right|^{(k+1) \rho}} \\
& \leqq \int_{-\delta}^{\delta} \frac{d s}{|s|^{(k+1) \rho}}=\frac{2 \delta^{1-(k+1) \rho}}{1-(k+1) \rho} .
\end{aligned}
$$

Hence the left hand side of (4.6) is estimated by a constant independent of $X=(x, y)$ in $\omega$. The inequality (4.4) with $\rho=0$ follows from (4.4) with $\rho>0$. The proof of (4.4) is complete. The proof of (4.4) is similar to that of (4.4).

In order to prove the inequality (4.5), we observe that

$$
\left|\frac{1}{w^{\prime}}-\frac{1}{w^{\prime \prime}}\right| \leqq \text { const }\left|w^{\prime}-w^{\prime \prime}\right|^{\rho}\left(\left|\frac{1}{w^{\prime}}\right|^{1+\rho}+\left|\frac{1}{w^{\prime \prime}}\right|^{1+\rho}\right)
$$

for every $\rho, 0 \leqq \rho \leqq 1$, and any two non-zero complex numbers $w^{\prime}$ and $w^{\prime \prime}$, as is easily verified. We shall set $w^{\prime}=e^{-1}\left(X^{\prime} ; X\right), w^{\prime \prime}=e^{-1}\left(X^{\prime \prime} ; X\right)$ in (4.7). Since

1) The use of the preparation theorem was suggested by Prof. Y. Kato. 


$$
w^{\prime}-w^{\prime \prime}=2 \pi\left[\int_{x}^{x \prime} b\left(z, y^{\prime}\right) d z-\int_{x}^{x \prime \prime} b\left(z, y^{\prime \prime}\right) d z+i\left(y^{\prime}-y^{\prime \prime}\right)\right]
$$

there is a constant $C$ such that $\left|w^{\prime}-w^{\prime \prime}\right| \leqq C\left|X^{\prime}-X^{\prime \prime}\right|$ if $X, X^{\prime}, X^{\prime \prime} \in \omega$, and this proves the inequality (4.5).

Proposition 4.2. For every $\rho, 0<\rho<\left(k_{p}(0,0)+1\right)^{-1}$, there is a constant $C$ such that

$$
\left\|E f i_{\rho} \leqq C\right\| f \|_{0}, \quad f \in C_{0}^{\infty}(\omega) .
$$

Here we have used the notation

$$
\|\| u \|_{s}^{2}=\int_{\omega}|u|^{2} d X+\iint_{\omega \times \omega}\left|u(X)-u\left(X^{\prime}\right)\right|^{2}\left|X-X^{\prime}\right|^{-2-2 s} d X d X^{\prime}
$$

Proof. By a well known inequality it follows from (4.4) and (4. 4) with $\rho=0$ that

$$
\int_{\omega}|u|^{2} d X \leqq M_{0}^{2}|| f \|_{0}^{2}
$$

where we have written $u=E f$.

Next we choose $\rho^{\prime}$ so that $\rho<\rho^{\prime}<\left(k_{p}(0,0)+1\right)^{-1}$. We obtain from (4.5) with $\rho=\rho^{\prime}$

$$
\begin{aligned}
\left|u\left(X^{\prime}\right)-u\left(X^{\prime \prime}\right)\right| \leqq N_{\rho^{\prime}} \mid X^{\prime}- & \left.X^{\prime \prime}\right|^{\rho^{\prime}} \int\left(\left|e\left(X^{\prime} ; X\right)\right|^{1+\rho^{\prime}}\right. \\
& \left.+\left|e\left(X^{\prime \prime} ; X\right)\right|^{1+\rho^{\prime}}\right)|f(X)| d X .
\end{aligned}
$$

If we set $U\left(X^{\prime}\right)=\int\left|e\left(X^{\prime} ; X\right)\right|^{1+\rho^{\prime}}|f(X)| d X$, we have

$$
\begin{aligned}
& \left|u\left(X^{\prime}\right)-u\left(X^{\prime \prime}\right)\right|^{2}\left|X^{\prime}-X^{\prime \prime}\right|^{-2-2 \rho} \\
& \quad \leqq 2 N_{\rho^{\prime}}^{2}\left(U^{2}\left(X^{\prime}\right)+U^{2}\left(X^{\prime \prime}\right)\right)\left|X^{\prime}-X^{\prime \prime}\right|^{-2+2\left(\rho^{\prime-\rho)}\right.} .
\end{aligned}
$$

Integrating over $\omega \times \omega$, we obtain

$$
\begin{aligned}
& \iint_{\omega \times \omega}\left|u\left(X^{\prime}\right)-u\left(X^{\prime \prime}\right)\right|^{2}\left|X^{\prime}-X^{\prime \prime}\right|^{-2-2 \rho} d X^{\prime} d X^{\prime \prime} \\
& \quad \leqq 4 N_{\rho}^{2} \iiint_{\omega \times \omega} U^{2}\left(X^{\prime}\right)\left|X^{\prime}-X^{\prime \prime}\right|^{-2+2(\rho \prime-\rho)} d X^{\prime} d X^{\prime \prime} \\
& \quad \leqq 4 N_{\rho^{\prime}}^{2 \prime} \int_{2 \omega}|X|^{-2+2\left(\rho^{\prime}-\rho\right)} d X \int_{\omega} U^{2}\left(X^{\prime}\right) d X^{\prime}
\end{aligned}
$$

Since $\rho^{\prime}>\rho$, the first integral is finite. From (4.4) and (4.4) with $\rho=\rho^{\prime}$ it follows that 


$$
\int_{\omega} U^{2}\left(X^{\prime}\right) d X^{\prime} \leqq M_{\rho}^{2}\|f\|_{0}^{2}
$$

This completes the proof of Proposition 4.2.

Proposition 4.3. $E$ is a left parametrix for $P$ in the following sense:

There exists a constant $C$ such that

$$
\|E P u-u\|_{\rho} \leqq C\|u\|_{0}, \quad u \in C_{0}^{\infty}(\omega) .
$$

Proof. We use the following identity

$$
d(v u(-i b d x+d y))=\left(v \cdot P u-{ }^{t} P v \cdot u\right) d x d y,
$$

where ${ }^{t} P$ is the formal transpose of $P$,

$$
{ }^{t} P=-D_{x}-i b D_{y}-i\left(D_{y} b\right) .
$$

Substituting $e\left(x^{\prime}, y^{\prime} ; x, y\right)$ for $v(x, y)$ and using Stokes formula, we obtain, for every $u \in C_{0}^{\infty}(\omega)$,

$$
\begin{aligned}
& \int e\left(X^{\prime} ; X\right) \cdot P u(X) d X=\int{ }^{t} P\left(X, D_{X}\right) e\left(X^{\prime} ; X\right) \cdot u(X) d X \\
& \quad-\lim _{\varepsilon \rightarrow 0} \int_{\Gamma_{\varepsilon}} e\left(X^{\prime} ; X\right) u(X)(-i b(x, y) d x+d y)
\end{aligned}
$$

where $\Gamma_{\varepsilon}$ is the contour of the square $\left|x-x^{\prime}\right| \leqq \varepsilon,\left|y-y^{\prime}\right| \leqq \varepsilon$.

First we shall show that the second term on the right hand side of (4.8) is equal to $u\left(X^{\prime}\right)$. Since

$$
\begin{aligned}
(2 \pi i)^{-1} d_{X} \log e^{-1}\left(X^{\prime} ; X\right) & =(2 \pi i)^{-1} e\left(X^{\prime} ; X\right) d_{X} e^{-1}\left(X^{\prime} ; X\right) \\
& =e\left(X^{\prime} ; X\right)\left(i b\left(x, y^{\prime}\right) d x-d y\right),
\end{aligned}
$$

we see that

$$
\begin{aligned}
& -e\left(X^{\prime} ; X\right) u(X)(-i b(x, y) d x+d y) \\
& =(2 \pi i)^{-1} u\left(X^{\prime}\right) d_{X} \log e^{-1}\left(X^{\prime} ; X\right)+e\left(X^{\prime} ; X\right)[i(u(x, y) b(x, y) \\
& \left.\quad-u\left(x^{\prime}, y^{\prime}\right) b\left(x, y^{\prime}\right)\right) d x-\left(u(x, y)-\left(u\left(x^{\prime}, y^{\prime}\right)\right) d y\right] .
\end{aligned}
$$

Since $\int_{x}^{x^{\prime}} b\left(z, y^{\prime}\right) d z$ is a strictly monotone decreasing function of $x$, when $X$ describes $\Gamma_{\varepsilon}$ in the positive direction, $e^{-1}\left(X^{\prime} ; X\right)$ describes a rectangle on the complex plane turning around the origin in the positive direction. Hence the integral over $\Gamma_{\varepsilon}$ of the first term on the right hand side of (4.9) is equal to $u\left(X^{\prime}\right)$. The integral of the 
second term can be estimated by a constant times

$$
\begin{aligned}
\varepsilon \int_{\Gamma_{\varepsilon}} \frac{d \sigma}{\left|x^{\prime}-x\right|^{k^{\prime}+1}+\left|y^{\prime}-y\right|} & =4 \varepsilon\left(\int_{0}^{\varepsilon} \frac{d s}{s^{k^{\prime}+1}+\varepsilon}+\int_{0}^{\varepsilon} \frac{d t}{\varepsilon^{k^{\prime}+1}+t}\right) \\
& \leqq 4 \varepsilon\left(1+\log \left(\varepsilon^{k^{\prime}+1}+\varepsilon\right)-\log \varepsilon^{k^{\prime}+1}\right),
\end{aligned}
$$

where $k^{\prime}=k_{p}\left(x^{\prime}, y^{\prime}\right),(d \sigma)^{2}=(d x)^{2}+(d y)^{2}$. Hence we see that, as $\varepsilon \rightarrow 0$, this integral tends to 0 .

Set $R u=E P u-u$. We have proved that

$$
R u\left(X^{\prime}\right)=\int{ }^{t} P\left(X, D_{X}\right) e\left(X^{\prime} ; X\right) \cdot u(X) d X, \quad u \in C_{0}^{\infty}(\omega)
$$

Now we have to prove that $\|R u\|_{\rho} \leqq C\|u\|_{0}, u \in C_{0}^{\infty}(\omega)$. The kernel of $R$ is

$$
\begin{aligned}
{ }^{t} P\left(X, D_{X}\right) e\left(X^{\prime} ; X\right)= & 2 \pi\left(b(x, y)-b\left(x, y^{\prime}\right)\right) e^{2}\left(X^{\prime} ; X\right) \\
& -i\left(D_{y} b\right)(x, y) e\left(X^{\prime} ; X\right) .
\end{aligned}
$$

We shall denote the first term on the right hand side of (4.10) by $r_{1}\left(X^{\prime} ; X\right)$ and the second by $r_{2}\left(X^{\prime} ; X\right)$. It follows from Proposition 4.2 that $\left\|\mid R_{2} u\right\|\left\|_{\rho} \leqq C\right\| u \|_{0}, u \in C_{0}^{\infty}(\omega)$.

We need an inequality of the type (4.5) for $r_{1}$. If we set $v^{\prime}=2 \pi\left(b(x, y)-b\left(x, y^{\prime}\right)\right), v^{\prime \prime}=2 \pi\left(b(x, y)-b\left(x, y^{\prime \prime}\right)\right), w^{\prime}=e^{-1}\left(X^{\prime} ; X\right)$, and $w^{\prime \prime}=e^{-1}\left(X^{\prime \prime} ; X\right)$, we see that if $X, X^{\prime}, X^{\prime \prime} \in \omega$

$$
\left|v^{\prime}\right| \leqq C\left|w^{\prime}\right|, \quad\left|v^{\prime \prime}\right| \leqq C\left|w^{\prime \prime}\right|, \quad\left|v^{\prime}-v^{\prime \prime}\right| \leqq C\left|w^{\prime}-w^{\prime \prime}\right| .
$$

Now we have

$$
\begin{aligned}
r_{1}\left(X^{\prime} ; X\right)-r_{1}\left(X^{\prime \prime} ; X\right) & =\frac{v^{\prime}}{w^{\prime 2}}-\frac{v^{\prime \prime}}{{w^{\prime \prime}}^{2}} \\
& =\left(w^{\prime \prime}-w^{\prime}\right) \frac{v^{\prime}}{w^{\prime}}\left(\frac{1}{v^{\prime} w^{\prime \prime}}+\frac{1}{w^{\prime \prime 2}}\right)+\left(v^{\prime}-v^{\prime \prime}\right) \frac{1}{{w^{\prime \prime 2}}^{2}}
\end{aligned}
$$

If $\left|w^{\prime}-w^{\prime \prime}\right| \leqq \frac{1}{2}\left|w^{\prime \prime}\right|$, then $\left|w^{\prime \prime}\right| \leqq 2\left|w^{\prime}\right|$ and

$$
\begin{aligned}
\left|r_{1}\left(X^{\prime} ; X\right)-r_{1}\left(X^{\prime \prime} ; X\right)\right| & \leqq 4 C \frac{\left|w^{\prime}-w^{\prime \prime}\right|}{\left|w^{\prime \prime}\right|^{2}} \\
& \leqq 2^{1+\rho} C\left|w^{\prime}-w^{\prime \prime}\right|^{\rho}\left|\frac{1}{w^{\prime \prime}}\right|^{1+\rho} .
\end{aligned}
$$

If $\left|w^{\prime}-w^{\prime \prime}\right| \geqq \frac{1}{2}\left|w^{\prime \prime}\right|$, then $\left|w^{\prime}\right| \leqq 3\left|w^{\prime}-w^{\prime \prime}\right|$ and 


$$
\begin{aligned}
& \left|r_{1}\left(X^{\prime} ; X\right)-r_{1}\left(X^{\prime \prime} ; X\right)\right| \leqq C\left(\frac{1}{\left|w^{\prime}\right|}+\frac{1}{\left|w^{\prime \prime}\right|}\right) \\
& \leqq C\left(3^{\rho}+2^{\rho}\right)\left|w^{\prime}-w^{\prime \prime}\right|^{\rho}\left(\left|\frac{1}{w^{\prime}}\right|^{1+\rho}+\left|\frac{1}{w^{\prime \prime}}\right|^{1+\rho}\right) .
\end{aligned}
$$

From (4.11) and (4.12), it follows that, for every $\rho, 0 \leqq \rho \leqq 1$, there is a constant $N_{\rho}^{\prime}$ such that if $X, X^{\prime} X^{\prime \prime} \in \omega$, we have

$$
\left|r_{1}\left(X^{\prime} ; X\right)-r_{1}\left(X^{\prime \prime} ; X\right)\right| \leqq N_{\rho}^{\prime}\left|X^{\prime}-X^{\prime \prime}\right|^{\rho}\left(\left|e\left(X^{\prime} ; X\right)\right|^{1+\rho}+\left|e\left(X^{\prime \prime} ; X\right)\right|^{1+\rho}\right) \text {. }
$$

Hence, by using the arguments given in Proposition 4.2,

$$
\left\|\mid R_{1} u\right\|_{\rho} \leqq C\|u\|_{0}, \quad u \in C_{0}^{\infty}(\omega) .
$$

This completes the proof of Proposition 4.3.

Since $\|u\| \|_{s}$ is equivalent to $\|u\|_{s}\left(0<s<1, u \in C_{0}^{\infty}\left(R^{2}\right)\right)[1, \S 2.6]$, it follows from Propositions 4.2 and 4.3 that for every $\rho, 0 \leqq \rho<$ $\left(k_{p}(0,0)+1\right)^{-1}$, there is a constant $C$ such that

$$
\|u\|_{\rho} \leqq C\left(\|P u\|_{0}+\|u\|_{0}\right), \quad u \in C_{0}^{\infty}(\omega) .
$$

This proves the sufficiency of the condition (A).

\section{$\S 5$. Necessity of the Condition (A)}

Let $P\left(x, y ; D_{x}, D_{y}\right)$ be a differential operator of order $m$ with simple real characteristics defined in a neighborhood $\Omega$ of $O$. Assume that $p(x, y ; \xi, \eta)$ has the form (2.2) and that, in addition, the coefficients of $p(x, y ; \xi, \eta)$ are analytic. Hence the coefficients $\lambda_{i}(x, y)$ are also analytic.

Suppose that the condition (A) does not hold for $p$. Then it follows from Proposition 2.2 that, for some $i$, (A) does not hold for $p_{i}$, that is, there is a point $\left(x_{0}, y_{0}\right)$ in $\Omega$ such that $k_{p_{i}}\left(x_{0}, y_{0}\right)$ is either finite and odd, or infinite. We proved in [5] that there exists then, in a neighborhood $\omega$ of $O$, a solution $u \in C^{1}(\omega)$ of $p_{i} u=0$ which is not in $C^{\infty}(\omega)$. Let $s_{0}$ be the supremum of $s$ such that $u \in H_{s}^{\text {loc }}(\omega)$. Since $u \notin C^{\infty}(\omega), s_{0}$ is finite. The operator $P$ can be written in the form

$$
P=Q p_{i}+R
$$

where $Q$ and $R$ are differential operators of order $m-1$. Since $p_{i} u=0$, we have $P u=R u$. Hence, for every $\rho>0, P u \in H_{s_{0}-(1 / 2) \rho-m+1}^{1 \text { loc }}(\omega)$ 
and $u \notin H_{s_{0}+(1 / 2) p}^{\text {loc }}(\omega)$. It follows from $\S 2,5^{\circ},(c)$ that an improving estimate does not hold for $P$. We have thus proved the necessity of the condition (A).

Remark. When there is a point $(x, y ; \xi, \eta)$ such that $k_{p}(x, y ; \xi, \eta)$ is finite and odd, then we need only assume that the coefficients of $p$ are $C^{\infty}$ functions [4].

\section{References}

[1] Hörmander, L., Linear Partial Differential Operators, Springer, 1963.

[2] - Pseudo-differential operators and non-elliptic boundary problems. Ann. of Math. 83 (1966), 129-209.

[3] Malgrange, B., Ideals of Differentiable Functions, Tata Institute of Fundamental Research, 1966.

[4] Nirenberg, L. and F. Treves, Solvability of a first order linear partial differential equation, Comm. Pure Appl. Math. 16 (1963), 331-351.

[5] Suzuki, H., Analytic-hypoelliptic differential operators of first order in two independent variables, J. Math. Soc. Japan, 16 (1964), 367-374.

Note added in proof (February 17, 1970): After this paper was accepted for publication, the author became aware of the fact that Ju. V. Egorov announced in Dokl. Akad. Nauk SSSR, Tom 188 (1699), No. 1, a solution of the problem for pseudodifferential operators in any number of independent variables. However, the method of proof in this paper is entirely different from his. 
